\title{
PANORAMA DOS GRUPOS DE PESQUISA EM POLÍTICAS PÚBLICAS DE ESPORTE E LAZER NO BRASIL PRESENTES NO DIRETÓRIO DE GRUPOS DE PESQUISA DO CNPQ
}

Recebido em: 26/07/2016

Aceito em: 18/01/2017

\author{
Andrea de Oliveira Barra \\ Centro Federal de Educação Tecnológica de Minas Gerais (CEFET/MG) \\ Nepomuceno - MG - Brasil \\ Sheila Aparecida Pereira dos Santos Silva \\ Universidade São Judas Tadeu \\ São Paulo - SP - Brasil \\ Maria Rachel Vitorino \\ Universidade Federal de Lavras (UFLA) \\ Lavras - MG - Brasil
}

RESUMO: O objetivo deste estudo foi localizar grupos de pesquisa em Políticas Públicas de Esporte e Lazer no Brasil, conhecer como se distribuem geograficamente no território nacional, e traçar um panorama do que vêm produzindo nesta linha de pesquisa. Para isso, realizou-se busca sistemática no Diretório de Grupos de Pesquisa do Conselho Nacional de Desenvolvimento Científico e Tecnológico, utilizando-se a técnica metodológica snowball, sobre a temática Políticas Públicas de Esporte e Lazer. Os grupos certificados estão distribuídos entre as regiões Nordeste $(n=12)$, Sudeste $(n=8)$, Sul $(n=5)$, Centro-Oeste $(n=4)$ e Norte $(n=1)$. Estes grupos desenvolvem 97 linhas de pesquisa, no entanto, apenas 33\% (n=32) são específicas de Políticas Públicas de Esporte e Lazer. Observou-se que houve um aumento, por ano, de 5,93\% no número de grupos de pesquisa, entre 1998 a 2015.

PALAVRAS CHAVE: Políticas Públicas. Esportes. Atividades de Lazer. Grupos de Pesquisa.

\section{OVERVIEW OF RESEARCH GROUPS IN PUBLIC POLICIES OF SPORT AND LEISURE IN BRAZIL PRESENTS THE DIRECTORY OF CNPQ RESEARCH GROUPS}

ABSTRACT: The aim of this study was to locate research groups on Leisure and Sports' Public Policies in Brazil, know how they are geographically distributed in the country and draw a picture of what has been produced in this research line. For this, we carried out a systematic search in the Research Groups Directory of the National Scientific and Technological Development Council, using the snowball methodological technique, on the theme Leisure and Sports' Public Policies. Certificated groups are 
distributed among the Northeast $(n=12)$, Southeast $(n=8)$, South $(n=5)$, Midwest $(n=4)$ and North $(n=1)$. These groups develop 97 lines of research, among these, however, only $33 \%(n=32)$ are specific in Leisure and Sports' Public Policies. It was observed that there was an increase per year of $5.93 \%$ in the number of research groups between 1998 and 2015.

KEYWORDS: Public Policies. Sports. Leisure Activities. Research Groups.

\section{Introdução}

As últimas décadas registraram o ressurgimento da importância do campo de conhecimento denominado políticas públicas (SOUZA, 2006).

Segundo Amabile (2012, p. 390), políticas públicas “[...] são decisões que envolvem questões de ordem pública com abrangência ampla, e que visam a satisfação do interesse de uma coletividade". Dito de outra maneira, políticas públicas são a totalidade de ações, metas e planos que os governos (nacionais, estaduais ou municipais) traçam para alcançar o bem-estar da sociedade e o interesse público, e possuem dupla função: instituir direitos sociais e exteriorizar a função planejadora do Estado.

À medida que os processos democráticos avançam, a população é convidada com mais frequência a expressar-se e a decidir, em conjunto com o poder público, a respeito das políticas públicas que atendam aos seus anseios e necessidades.

$\mathrm{Na}$ área da Educação Física, políticas públicas tem sido pauta de discussões entre estudiosos. Como exemplo, podem-se mencionar os trabalhos de Linhales; Pereira Filho (1999); Manhães (2002); Santos; Batista e Araújo (2007); Linhales (2008); Húngaro et al. (2009); Werle (2010); Starepravo; Souza; Marchi Jr (2011, 2012, 2013); Zotovici et al. (2013). 
Entre os autores, parece consensual que essas discussões necessitam de evidências empíricas, cientificamente sistematizadas, que justifiquem os investimentos nas políticas públicas de Esporte e Lazer.

A maioria das pesquisas sobre políticas públicas de Esporte e Lazer estão inseridas no contexto das análises nas estruturas e instituições, que contribuem para a elaboração de investigações de natureza descritiva, conforme apontam Amaral; Ribeiro e Silva (2014), embora já se possam notar pesquisas que mesclam microanálises com análises macroestruturais, o que parece ser desejável nos apontamentos de Frey (2000).

Outra tendência das políticas públicas de Esporte e Lazer no Brasil está relacionada ao crescimento da agenda exemplificada pelos megaeventos esportivos, com destaque para a Copa do Mundo da FIFA e os Jogos Olímpicos que serão realizados no Rio de Janeiro em 2016.

Tem-se hoje, grande quantidade de perspectivas teóricas e metodológicas disponíveis, marcadas não apenas por diferentes escolas de pensamento, e pelos contextos políticos e socioculturais em que foram produzidos, mas também, por estilos intelectuais muito diversos. Assim, sem esgotar o tema, é importante incluir o contexto acadêmico nessa análise, começando por localizar e identificar os grupos de pesquisa no Brasil, que têm como objetivo estudar Políticas Públicas de Esporte e Lazer.

Uma das formas de identificar quais são os grupos de pesquisa no Brasil, que têm como objetivo estudar políticas públicas de Esporte e Lazer é por meio do Diretório de Grupos de Pesquisa do Conselho Nacional de Desenvolvimento Científico e Tecnológico (DGP/CNPq).

O DGP/CNPq constitui um inventário dos grupos de pesquisa científica e tecnológica em atividade no país, cadastram recursos humanos constituintes dos grupos 
(pesquisadores, estudantes e técnicos), as linhas de pesquisa em andamento, as especialidades do conhecimento, os setores de aplicação envolvidos, a produção científica, tecnológica e artística, e as parcerias estabelecidas entre os grupos e as instituições, sobretudo com as empresas do setor produtivo (BRASIL, 2016).

Sua análise permite descrever os limites e o perfil geral da atividade científicotecnológica no Brasil. Este diretório tem, portanto, importante papel na preservação da memória da atividade científico-tecnológica no Brasil, e constitui ferramenta para interação/troca de informações. Sua base de dados traz informações sobre os grupos, tais como sua localização e produções científicas nos últimos anos.

Para localizar os Grupos de Pesquisa em Políticas Públicas de Esporte e Lazer no Brasil (GPPPEL), que possuem linhas de pesquisa específicas de Políticas Públicas de Esporte e Lazer (PPEL), foi necessário identificar o surgimento destes grupos, sua distribuição no país e sua constituição. Além disso, esses dados podem possibilitar o apontamento de um panorama da produção científica, além da titulação dos pesquisadores das PPEL. Segundo Starepravo (2007, 2011); Starepravo e Mezzadri (2007), são escassas e parecem sofrer de certa falta de consistência teórica, as informações sobre a atuação de grupos de pesquisas na área de PPEL, nas distintas regiões brasileiras.

Mapear grupos de pesquisa com linhas de pesquisa específicas de PPEL, bem como sua produção, é importante para compreender e refletir sobre o fazer pesquisa, produzir e divulgar conhecimento, oferecendo subsídios para as diretrizes e políticas institucionais, o que resulta em novos conhecimentos para a sociedade. A partir deste tipo de conhecimento, talvez seja possível traçar estratégias para reforçar a capacidade 
de produção de pesquisas voltadas ao desenvolvimento de políticas públicas, bem como incentivar a participação de estudantes em sua realização, por exemplo.

Neste contexto, traçou-se como objetivo de estudo, localizar os GPPPEL no Brasil, que possuem linhas de pesquisa específicas de PPEL, conhecer como se distribuem geograficamente no território nacional, e traçar um panorama do que vêm produzindo.

\section{Aspectos Metodológicos}

Este artigo é fruto de um estudo sistemático e descritivo. Segundo Sampaio e Mancini (2007, p. 84) "[...] métodos sistemáticos são usados para evitar viés e possibilitar uma análise mais objetiva dos resultados, facilitando uma síntese conclusiva".

O estudo foi realizado na base de dados do Diretório de Grupos de Pesquisa do CNPq (DGP/CNPq) sobre a temática PPEL, durante o mês de maio de 2016. Para tanto, utilizou-se de forma adaptada, a técnica metodológica snowball, também conhecida como snowball sampling (BIERNACKI; WALDORF, 1981), que se baseia em uma forma de amostra não probabilística, utilizada geralmente em pesquisas sociais, que analisa as ligações entre membros com características de interesse em comum.

As buscas ocorreram por meio das expressões: "Políticas Públicas de Esporte e Lazer", "Políticas Públicas de Esporte, Lazer", "Políticas Públicas de Esporte", “Políticas Públicas para o Esporte”, "Políticas Públicas para o Esporte e Lazer" e “Políticas de Esporte e Lazer". Tais expressões permitiram identificar grupos associados aos temas, ou seja, tais termos de busca sobre a área pesquisada foram aplicados aos nomes dos grupos, aos nomes da linha de pesquisa e às palavras-chaves da linha de 
pesquisa. Essa busca inicial, por sua vez, fundamentou a realização de novos levantamentos de grupos, até que fosse alcançado o chamado "ponto de saturação", no qual os conteúdos interligados passaram a se repetir, sem acréscimo de novas informações.

Nos dados que resultaram da busca, foi identificado o ano de formação do grupo (até dezembro de 2015), sua localização geográfica, o número de pesquisadores da linha de pesquisa em PPEL e a titulação dos mesmos, além de traçarmos um panorama das formas de veiculação da produção científica, dos grupos nas linhas de pesquisa específicas em PPEL. Para tanto, foram considerados os tipos de produção científica, sem análise de sua temática, tomando como base os "indicadores de produção" de cada pesquisador cadastrado, considerando o período compreendido entre o ano de formação do grupo e o ano de 2015, nas linhas de pesquisa diretamente ligadas às PPEL. Para ter acesso aos indicadores de produção, buscou-se, dentro do GPPPEL, a linha de pesquisa em PPEL, logo após, buscou-se pelos Recursos Humanos/Pesquisadores relacionados à linha, e em seguida, para cada pesquisador cadastrado, buscou-se o item 'visualizar espelho do pesquisador'.

Segundo Brasil (2016), a certificação dos grupos de pesquisa é de responsabilidade dos dirigentes das atividades de pesquisa da instituição, a que o líder do grupo está vinculado. A certificação do GPPPEL pelos dirigentes institucionais de pesquisa foi adotada como critério de inclusão.

Neste estudo, não se analisou a Rede Cedes, pois já houve pesquisas neste sentido, como destacam as pesquisas de Schwartz et al.(2010) e Starepravo (2011).

As informações recolhidas dos pesquisadores pertencentes às linhas de pesquisa específicas de PPEL dos grupos foram tabuladas, e para a apresentação dos resultados, 
foram utilizados procedimentos da estatística descritiva, por meio de frequência absoluta e relativa, além da aplicação do Teste t (FIGURA 3).

\section{Resultados}

Foram identificados 30 (trinta) grupos de pesquisa cadastrados em três áreas de conhecimento, sendo 21 (vinte e um) em Ciências da Saúde (Educação Física, n=21), 8 (oito) em Ciências Humanas (Ciência Política, n=2, Educação, n=4 e Sociologia, n=2) e 1 (um) em Ciências Sociais Aplicadas (Administração, n=1). Os GPPPEL encontram-se distribuídos em 25 (vinte e cinco) instituições públicas e 5 (cinco) privadas.

O número total dos GPPPEL está distribuído em 13 (treze) estados brasileiros: Bahia, Distrito Federal, Goiás, Maranhão, Mato Grosso, Mato Grosso do Sul, Minas Gerais, Paraná, Pernambuco, Rio de Janeiro, Rio Grande do Sul, Roraima, São Paulo, abrangendo todas as regiões do Brasil (FIGURA 1).

Figura 1 - Distribuição geográfica dos GPPPEL no Brasil, reconhecidos oficialmente até dezembro de 2015.

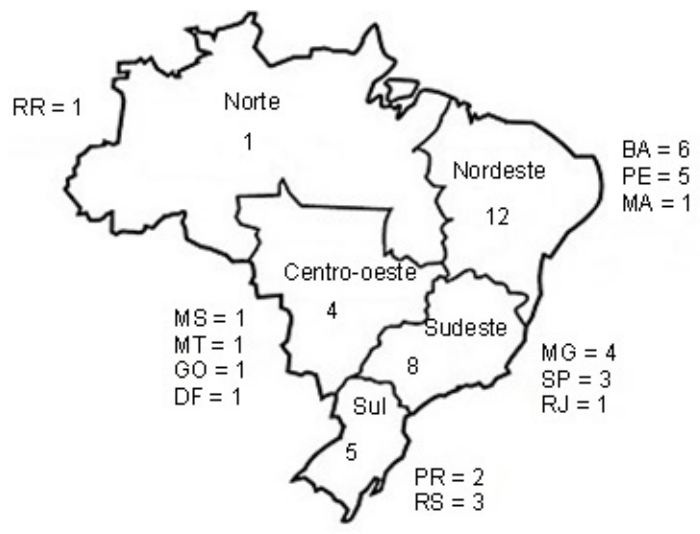

Fonte: dados da pesquisa (frequência absoluta por estado e região) 
A Figura 2 mostra uma linha do tempo na qual é possível visualizar a progressão anual do número de GPPPEL, levando em consideração a área de conhecimento predominante do grupo.

Figura 2 - Progressão anual do número de GPPPEL em relação à área de conhecimento.

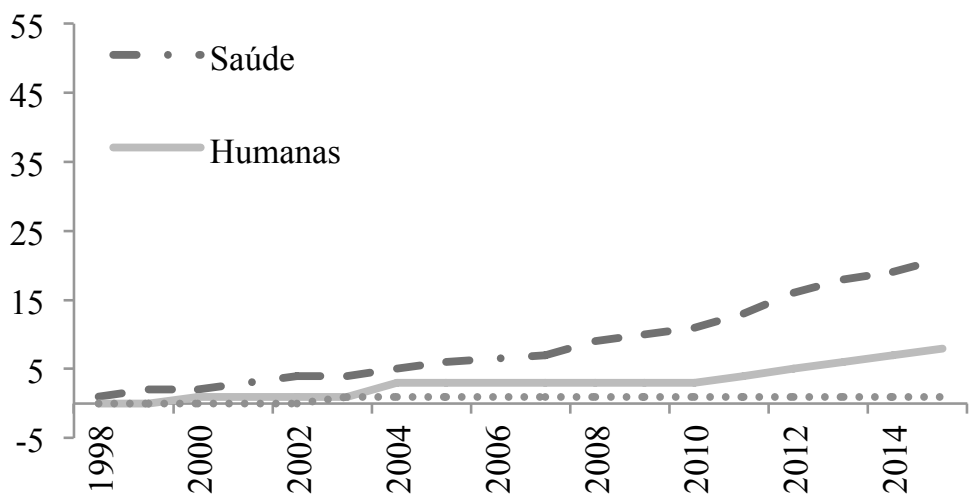

Fonte: dados da pesquisa (frequência absoluta por área do conhecimento)

O GPPPEL mais antigo foi formado em 1998.

A Figura 3 mostra um comportamento linear entre o número de grupos e ano de criação dos mesmos, cujo modelo é dado por: (1) $\mathrm{y}=1,779 \mathrm{x}-3,426$.

Por meio do Teste t verificou-se que os parâmetros do modelo de regressão expresso em (1) foram significativos estatisticamente, como consta na (TABELA 1). Uma interpretação para a expressão (1), é que a cada ano está havendo um acréscimo médio de 1.77941 grupos.

Tabela 1 - Análise de regressão

\begin{tabular}{|c|c|c|c|c|}
\hline & Estimativa & Erro padrão & Teste t & P - valor \\
\hline $\mathrm{a}$ & -3.42647 & 0.94694 & -3.618 & $0.00253 *$ \\
\hline $\mathrm{b}$ & 1.77941 & 0.09241 & 19.255 & $<0.0001 *$ \\
\hline
\end{tabular}

Fonte: dados da pesquisa. 
Observou-se também pelo coeficiente de regressão, $\mathrm{R}^{2}=0,961$, que houve um autoajuste entre as variáveis envolvidas (ano de criação $=\mathrm{a}, \mathrm{n}^{\mathrm{o}}$ de grupos $=\mathrm{b}$;), podendo ser observado na (FIGURA 3).

Figura 3 - Progressão do número de GPPPEL com linha de tendência polinomial.

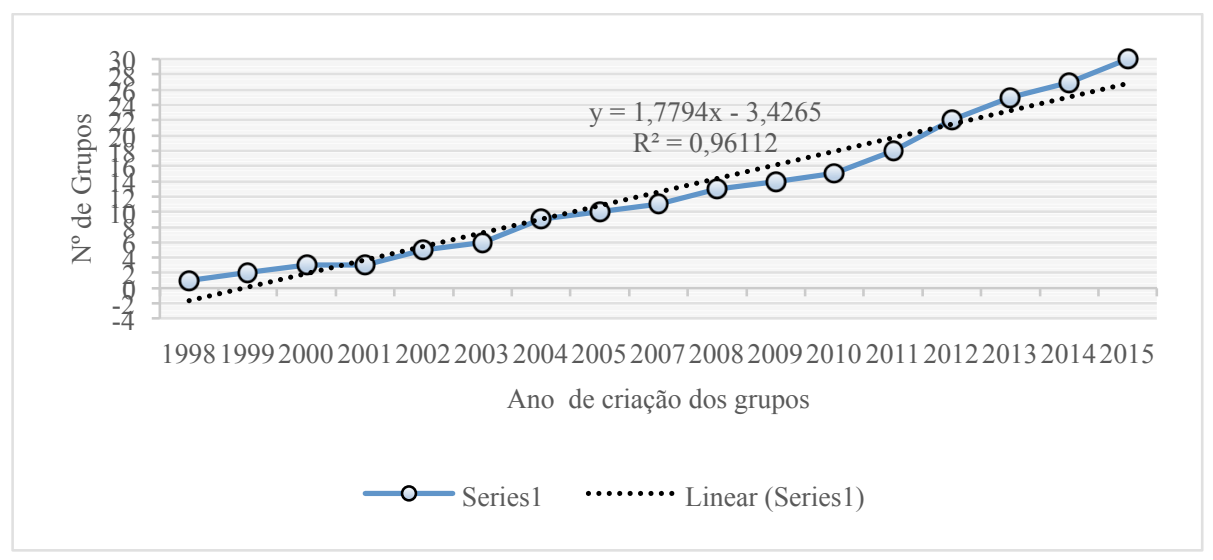

Fonte: dados da pesquisa ( ${ }^{\circ}$ de grupos acumulados)

A partir destes dados iniciais, optou-se por fazer a análise do perfil dos pesquisadores e do panorama dos formatos de veiculação de suas publicações, levando em consideração as linhas de pesquisa específicas de PPEL. Os trinta GPPPEL identificados desenvolvem (97) noventa e sete linhas de pesquisa. No entanto, apenas $33 \%$ (n=32) são específicas de PPEL.

Considerando os pesquisadores cadastrados nestas 32 (trinta e duas) linhas de pesquisa, 71 (setenta e um) são doutores, 24 (vinte e quatro) são mestres, 8 (oito) são especialistas e 7 (sete) são graduados. Quando comparadas as titulações dos pesquisadores do tema, por região, a região Nordeste é a que conta com maior número de doutores (FIGURA 4). 
Figura 4 - Titulação acadêmica dos pesquisadores das linhas de pesquisa específicas em PPEL dos GPPPEL brasileiros por região.

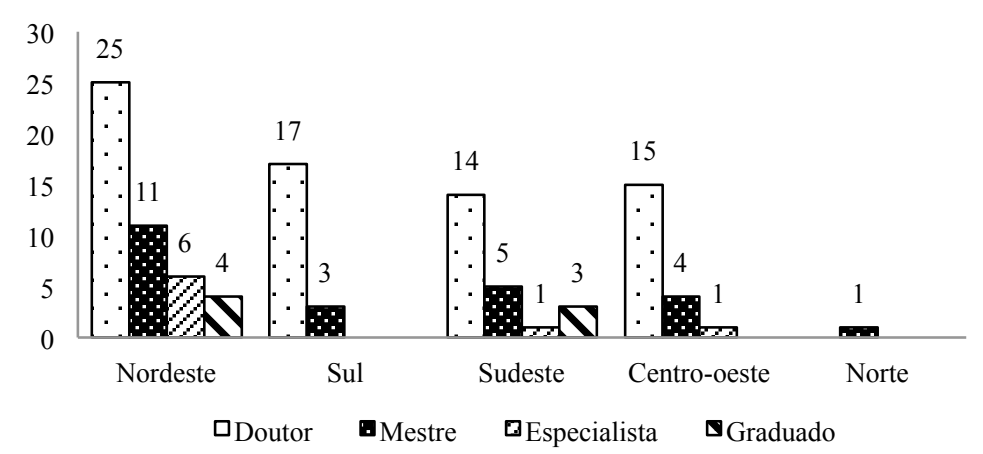

Fonte: dados da pesquisa (frequência absoluta da titulação dos pesquisadores).

Traçando um panorama da produção científica do que vêm produzindo os GPPPEL na linha de pesquisa em PPEL, e das formas escolhidas para veicular essa produção, esse panorama não conseguiu apurar se tal produção é específica da linha de pesquisa em PPEL, pois, o que se indica no DGP/CNPq são os totais de produção do grupo de pesquisa, sem discriminar a produção por linha de pesquisa desses grupos que, via de regra, possuem mais do que uma. Por meio dos indicadores de produção dos pesquisadores foram contabilizados os artigos publicados em periódicos indexados, os trabalhos completos publicados em anais de congressos, os livros publicados ou organizados, e os capítulos de livros, a partir do ano de criação do grupo de pesquisa (TABELA 2).

Tabela 2 - Formato de veiculação da produção científica dos grupos nas linhas de pesquisa específicas em PPEL, distribuída entre as regiões.

\begin{tabular}{|c|c|c|c|c|c|c|}
\hline $\begin{array}{c}\text { Formato de veiculação da } \\
\text { produção científica/Região }\end{array}$ & NE & S & SE & CO & N & Total \\
\hline $\begin{array}{c}\text { Artigos completos em } \\
\text { periódicos }\end{array}$ & 319 & 248 & 182 & 163 & 7 & 919 \\
\hline $\begin{array}{c}\text { Trabalhos completos } \\
\text { publicados em anais de } \\
\text { congressos }\end{array}$ & 324 & 192 & 76 & 79 & 0 & 671 \\
\hline $\begin{array}{c}\text { Capítulos de livros publicados } \\
\text { Livros }\end{array}$ & 189 & 76 & 112 & 62 & 1 & 440 \\
\hline Total & 35 & 15 & 23 & 10 & 0 & 83 \\
\hline
\end{tabular}

Fonte: dados da pesquisa (frequência absoluta por regiões - nordeste (NE), sul (S), sudeste (SE), norte $(\mathrm{N})$ e Centro-oeste $(\mathrm{CO}))$. 
De acordo com a Tabela 2, os artigos publicados em periódicos, baseado na frequência relativa, correspondem a 43,5\% $(n=919)$ da produção científica dos pesquisadores. Estes 919 (novecentos e dezenove) trabalhos representam 34,7\% $(n=319)$ da produção científica na região Nordeste, $27,0 \%(n=248)$ na região Sul, 19,8\% $(\mathrm{n}=182)$ na região Sudeste, $17,7(\mathrm{n}=163)$ na região Centro-Oeste, e $0,8(\mathrm{n}=7)$ na região Norte.

Na Tabela 3, são apresentados os recursos humanos dos GPPPEL com o intuito de mostrar como os grupos se organizam.

Tabela 3 - Recursos humanos dos GPPPEL no Brasil nas linhas de pesquisa específicas em PPEL, distribuídos por região.

\begin{tabular}{|c|c|c|c|c|}
\hline Região & Pesquisadores & Estudantes & Técnicos & Total \\
\hline Nordeste & 46 & 96 & 6 & 148 \\
\hline Sul & 20 & 38 & 0 & 58 \\
\hline Centro-oeste & 20 & 17 & 0 & 37 \\
\hline Sudeste & 23 & 12 & 1 & 36 \\
\hline Norte & 1 & 0 & 0 & 1 \\
\hline TOTAL & $\mathbf{1 1 0}$ & $\mathbf{1 6 3}$ & $\mathbf{7}$ & $\mathbf{2 8 0}$ \\
\hline
\end{tabular}

Fonte: dados da pesquisa (frequência absoluta dos recursos humanos por região).

Em relação à distribuição dos membros dos grupos, a região Nordeste concentra o maior número de integrantes, $52,86 \%(\mathrm{n}=148)$, seguida pelas regiões Sul, com $20,71 \%$ $(n=58)$, Centro-Oeste, com 13,21\% $(n=37)$, Sudeste, com 12,86\% $(n=36)$, e Norte, com $0,36 \%(n=1)$.

\section{Discussão}

Segundo o Censo $2014^{1}$ divulgado pelo Ministério da Ciência e Tecnologia, de modo geral,

O Nordeste aumentou sua participação no total dos grupos de $18,3 \%$ para $20,4 \%$ entre 2010 e 2014, aproximando-se do Sul, estável na casa

\footnotetext{
1 Em 2014, a base censitária foi composta por grupos certificados, existentes na base corrente do Diretório de Grupos do CNPq em 30 de dezembro de 2014.
} 
dos 22\%. Em 2004, a participação do Nordeste era de 14,2\% (BRASIL, 2014, s/p).

Os dados apresentados na Figura 1, em relação à região Nordeste, corroboram com Brasil (2014), no que diz respeito ao aumento da participação desta região em relação ao total de GPPPEL (n-12). Esta região é a que conta com o maior número de estados.

Foi possível perceber que esta região apresenta o maior número de doutores, pesquisando a temática das PPEL em relação aos outros estados. Algumas possíveis razões para o desenvolvimento dos GPPPEL e, consequentemente, no número de doutores nesta região, podem ser: criação e consolidação de cursos de pós-graduação; formação de massa crítica; maior investimento em pesquisa; aparato das tecnologias emergentes; consolidação das linhas de pesquisa nos programas de pós-graduação. Tais razões podem refletir positivamente na produção científica dos integrantes das linhas de pesquisa em PPEL, no entanto, para que haja comprovação da relação entre estas razões apontadas e os resultados apresentados, será necessário realizar outras pesquisas sobre o assunto.

Ainda segundo o Censo 2014, de maneira geral, “[...] o crescimento do número de grupos cadastrados em 2014 em relação a 2002 foi de 134\%" em todo o Brasil. Traçando um paralelo em relação aos GPPPEL, observou-se que houve um aumento, por ano, de 5,93\% no número de grupos, desde 1998 até 2015, o que significa um acréscimo, em média, de 1,77941 de GPPPEL, por ano, no Brasil.

O início da formação dos GPPPEL ocorreu na década de 90, paralelamente à abertura democrática sobre políticas públicas direcionadas ao esporte (STAREPRAVO, 2011), porém, a consolidação destes grupos aconteceu a partir da organização e 
sistematização efetuada pelo CNPq. Isso destaca a importância da continuidade do incentivo aos GPPPEL por meio do fomento à criação de linhas de pesquisa em PPEL, considerando que o CNPq exerce papel relevante.

O aumento no número de GPPPEL nos últimos 5 anos pode ser justificado, segundo Amaral; Ribeiro e Silva (2014), pelas mudanças nos investimentos na área das PPEL no Brasil.

Parece, após a análise da progressão do número de GPPPEL, que as áreas do conhecimento que se mostram mais envolvidas com as discussões acadêmicas voltadas para as PPEL são as Ciências da Saúde e as Ciências Humanas. As Ciências da Saúde talvez por buscarem a conjunção entre teoria e prática, visando a formação de um profissional que integre conhecimentos de diferentes áreas, e tenha uma visão de mundo crítica e reflexiva. As Ciências Humanas, por tratarem de aspectos filosófico-históricosociais da vida humana e, nessa abrangência, consta a Ciência Política como uma área do conhecimento, que com base em estudos sobre a estrutura e a dinâmica social, coloca em pauta aspectos que são fundamentais para a compreensão dos fenômenos políticos e das instituições pelas quais uma sociedade se governa.

Entende-se que os temas Esporte e Lazer, enquanto políticas públicas, podem ser tratados tanto pela Educação Física/Ciência da Saúde, como por outras disciplinas situadas em outras áreas classificadas como Ciência Política, Sociologia, Educação/Ciências Humanas ou Administração/Ciências Sociais Aplicadas. Esse interesse de diferentes áreas pela temática foi constatado nesta pesquisa, como também sua crescente abordagem de forma interdisciplinar.

Considerando as formas de veiculação das produções científicas, sem análise da temática, a partir do item 'indicadores de produção' de cada pesquisador cadastrado, o 
panorama verificado nos mostra que a publicação de artigos científicos é o meio mais utilizado para a disseminação das pesquisas. Vale ressaltar as colocações de Silva et al. (2006, p. 172), de que a produção científica não se compõe somente da realização da pesquisa em si,

[...] mas, principalmente, pela comunicação dos resultados desta pesquisa. O processo de comunicação é de suma importância, pois a divulgação dos resultados obtidos é o primeiro passo para que o conhecimento científico adquira confiabilidade e os autores credibilidade e prestígio (SILVA et al. 2006, p. 172).

Ainda nesta perspectiva, autores como Danuello e Guimarães (2005) consideram que a produção científica e a sua disseminação é um importante indicador de competência, reconhecido internacionalmente, no que tange ao "[...] trabalho original publicado numa revista considerada de boa qualidade pela comunidade científica ( $\mathrm{p}$. 158)".

Evidências epistemológicas confirmam que a produção de conhecimento das PPEL no Brasil tem uma história recente, como nos apontam: Húngaro et al. (2009); Werle (2010); Starepravo; Souza; Marchi Jr (2011, 2012, 2013); Zotovici et al. (2013); Mezzadri (2014) e que vem ganhando força e destaque advindos da grande contribuição de vários autores como: Alcyane Marinho, Ana Claudia Porfírio Couto, Celi Nelza Z. Taffarel, Christianne Luce Gomes, Edson Marcelo Húngaro, Hélder Ferreira Isayama, Fernando Augusto Starepravo, Fernando Marinho Mezzadri, Leila Mirtes Santos de Magalhães Pinto, Lino Castellani Filho, Nelson Carvalho Marcellino, Ricardo Ricci Uvinha, Marco Paulo Stigger, Pedro Fernando Avalone de Athayde, Gisele Maria Schwartz, Victor Andrade de Melo, Wanderley Marchi Júnior, e outros já mencionados neste estudo. 
Conforme mencionado, os trinta GPPPEL encontram-se inseridos nas instituições de ensino da seguinte forma: vinte e cinco instituições públicas e cinco privadas. Proporção semelhante foi observada no panorama da produção científica traçado, onde se observa que dos 919 artigos científicos publicados em revistas nacionais e internacionais, $98,15 \%$ (n=902) são provenientes de instituições públicas e $1,85 \%(n=17)$ de instituições privadas. Estes dados corroboram o levantamento feito por Silva (2001) que mostra que $94,7 \%$ da produção científica do país, na forma de trabalhos publicados em revistas nacionais e internacionais, vêm de universidades públicas, o que parece evidenciar a carência do exercício da publicação científica em universidade privadas em relação à temática, visando a inserção internacional de sua produção e a consequente visibilidade por outros países.

Neste sentido, o levantamento feito em relação às publicações dos pesquisadores das linhas de pesquisa de PPEL, se faz pertinente quando se indaga se a produção científica tem sido um dos subsídios à formulação e implementação das PPEL. Não há como dar resposta afirmativa ou negativa, mas essas inquietações podem levar a compreender os espaços sociais envolvidos, o subcampo científico/acadêmico no qual se produz conhecimento sobre PPEL; e o subcampo político/burocrático no qual se formulam e implementam as políticas públicas e as possibilidades de aproximação entre eles, já que é isso que se espera da pesquisa social, que ela seja aplicável e viável (STAREPRAVO, 2013).

Conforme afirma Starepravo (2011, p. 314), “[...] o subcampo científico/acadêmico das Políticas Públicas de Esporte e Lazer se apresenta como um espaço social que ainda passa por um processo de consolidação". 
Contudo, percebe-se que o Brasil caminha em busca da excelência em nível de produção de pesquisas, sejam elas em PPEL ou em outras áreas, tendo em vista a organização da investigação por meio de grupos de pesquisa, linhas de pesquisa, o próprio $\mathrm{DGP} / \mathrm{CNPq}$ e centros de investigação, assim como a constituição de redes de informação disponibilizadas na internet em várias áreas do conhecimento, como, por exemplo: Rede Cedes, Centro Esportivo Virtual, dentre outras.

A produção sobre o tema ainda pode ter relação direta com a formação universitária, em especial com os Programas de Pós-Graduação Stricto Sensu, que fomentam a formação de pesquisadores crítico-reflexivos, e qualificados para produzir novos conhecimentos.

Apoiados na ideia de Teixeira (2005) concorda-se que a expansão da produção de pesquisas é resultado de ações que visaram a formação e aperfeiçoamento de pesquisadores. Constatar que os estudantes formam a maioria dos integrantes das linhas de pesquisa em PPEL dos grupos analisados mostra que no Brasil há ações estimuladoras para formação de novos pesquisadores e produtores de conhecimento.

\section{Considerações Finais}

Uma área de estudo é composta por um corpo de conhecimento de literatura relativo à teoria e à prática, por profissionais que formam outros profissionais, por aqueles que desenvolvem pesquisa dentro e fora dos grupos de pesquisa, pelos que atuam na prática, por organizações profisssionais dedicadas ao avanço da área, pela formação profissional e pela credibilidade que ela conquista perante a sociedade (PITTS, 2001). 
O presente estudo mostrou que a discussão em grupos de pesquisa sobre a temática das PPEL está disseminada por todo o Brasil, já que há pelo menos um GPPPEL em cada região do país.

Observou-se também, que houve uma evolução substancial na criação dos GPPPEL, em um período de dezessete anos. Este aumento expressivo é um dado positivo, já que um grupo de pesquisa colabora com a sociedade ao proporcionar uma formação sólida aos graduandos, ancorado no ensino, pesquisa e extensão, sem descuidar do aparato teórico conceitual e empírico, e busca a formação de cidadãos comprometidos com a transformação social, em direção a uma formação cidadã. Desta maneira, buscam imprimir uma educação emancipadora, que respeita os limites e as possibilidades inscritas pela realidade (GUZZO e EUZEBIOS FILHO, 2005).

Outro destaque deste estudo é a importante participação de pesquisadores de outras áreas do conhecimento, que não das Ciências da Saúde, nos GPPEL, pois as novas fronteiras com que a ciência se depara indicam que o conhecimento específico de uma área de conhecimento não é suficiente para entender e atender a complexidade dos fenômenos estudados. Assim, torna-se indispensável transpassar as fronteiras interdisciplinares para enriquecer cada disciplina, e o conhecimento humano como um todo, com pontos de vista provenientes de diferentes horizontes. Isso nos remete a uma ideia de ressignificação das ciências, no sentido de agregar referenciais das áreas humanas e sociais, que contribuem, sobremaneira, para a ampliação do entendimento dos fenômenos da Educação Física, Esporte e Lazer, assim como para o amadurecimento da área.

Destaca-se que a criação de novos GPPPEL e suas respectivas linhas de pesquisa de PPEL é de suma importância no sentido de fomentar a consciência crítica dos 
estudantes e pesquisadores, objetivando a qualificação dos acadêmicos para que possam intervir nas questões sociais, de forma a melhorar as condições de participação nas PPEL. Espera-se que tais grupos estejam conscientes da sua importância na disseminação da ciência como participantes de um projeto coletivo, produção voltada a subsidiar proposições e avaliações de PPEL que contribuam para a transformação social.

Mais que um espaço institucionalizado, os grupos de pesquisa devem comprometer-se efetivamente com o desenvolvimento das PPEL, contribuindo para o avanço da construção do conhecimento e na construção de um espaço de reflexão teórico-prático sólido.

Por fim, a limitação deste estudo encontra-se no fato de ser um recorte da realidade dos GPPPEL nas linhas específicas de PPEL, nos anos de 1998-2015. Assim, destaca-se a necessidade da realização de outros estudos sobre essa temática no Brasil, enfocando as linhas de investigação estruturadas, a produção científica específica em PPEL desenvolvida nestes grupos, bem como a possibilidade de realizar um estudo, agora contando com os dados do Censo de 2015 do CNPq, a fim de traçar um paralelo de avaliação do processo de desenvolvimento do setor de PPEL.

\section{REFERÊNCIAS}

AMABILE, A. E. N. Políticas Públicas. In: CASTRO, C. L. F.; GONTIJO, C. R. B.; AMABILE, A. E. N. (Org.)Dicionário de Políticas Públicas. Barbacena: EdUEMG, 2012, p. 390-391. Disponível em: $<$ https://pt.calameo.com/read/0016339049620b36a7dac $>$ Acesso em: 01 jul. 2016.

AMARAL, S. C. F.; RIBEIRO, O. C. F.; SILVA, D. S. Produção científico-acadêmica em políticas públicas de Esporte e Lazer no Brasil. Motrivivência, v. 26, n.42, p. 27-40, Jun, $2014 . \quad$ Disponível em: http://periodicos.ufsc.br/index.php/motrivivencia/article/view/21758042.2014v26n42p27/27264 Acesso em: 01 jul. 2016. 
BIERNACKI, P.; WALDORF, D. Snowball sampling: problems and techniques of chain referral sampling. Sociological Methods \& Research, v. 10, n. 2, p. 141-163, Nov., 1981.

BRASIL. Ministério de Ciências e Tecnologia; Conselho Nacional de Desenvolvimento Científico e Tecnológico (CNPq). Diretório dos Grupos de Pesquisa no Brasil: O Diretório [Internet]. Brasília: Ministério da Ciência e Tecnologia; Atualizada jul. 2016; Disponível em: < https://lattes.cnpq.br/web/dgp/home>. Acesso em: 01 jul. 2016.

. Ministério de Ciências e Tecnologia; Conselho Nacional de Desenvolvimento Científico e Tecnológico $(\mathrm{CNPq})$. Grupos de Pesquisa: Censos [Internet]. Brasília: Ministério da Ciência e Tecnologia, 2014. Atualizada jul. 2016 Disponível em: $<$ https://lattes.cnpq.br/web/dgp/censo-atual $>$ Acesso em: 01 jul. 2016.

DANUELlO, J. C.; GUIMARÃES, J. A. C. Produção científica Docente em tratamento temático da informação nos cursos de biblioteconomia do Mercosul: uma análise preliminar. Transinformação. Campinas, v. 17, n. 2, p. 153-168, Maio/Ago., 2005. Disponível em: https://www.scielo.br/pdf/tinf/v17n2/04.pdf Acesso em: 05 jul. 2016.

FREY, K. Políticas públicas: um debate conceitual e reflexões referentes à prática da análise de políticas públicas no Brasil. Planejamento e Políticas Públicas, v. 21, p. 211-259, Jun., 2000. Disponível em: https://www.en.ipea.gov.br/ppp/index.php/PPP/article/viewFile/89/158 Acesso em: 05 jul. 2016.

GUZZO, R. S. Lobo; EUZEBIOS FILHO, A. Desigualdade social e sistema educacional brasileiro: a urgência da educação emancipadora. Escritos Sobre Educação. v. 4, n.2, p.39-48, Dez, 2005. Disponível em: https://pepsic.bvsalud.org/scielo.php?script=sci_arttext\&pid=S167798432005000200005 Acesso em: 05 jul. 2016.

HÚNGARO, E. M.; OLIVEIRA, B. A.; CUSTÓDIO, M. L.; DAMASCENO, L. G. Balanço inicial da produção do GTT de políticas públicas do CBCE (1997-2005): avanços, ausências e perspectivas. In: HÚNGARO, E. M.; SOUSA, W. L. Lino de (Org.). Cultura, educação, lazer e esporte: fundamentos, balanços e anotações críticas. Santo André: Alpharrabio, 2009, p. 93-124.

LINHALES, Meily Assbú; PEREIRA FILHO, José Ribamar. Intervenção, conhecimento e mudança: a Educação Física, o esporte e o lazer as políticas públicas. In: GOELLNER, Silvana Vilodre. Educação Física / Ciências do Esporte: intervenção e conhecimento. Campinas: Autores Associados, 1999.

et al. Esporte e Lazer na Grande-MH: por onde caminham as gestões públicas? In: ISAYAMA, Helder Ferreira; LINHALES, Meily Assbú (Org.). Avaliação de políticas e políticas de avaliação: questões para o esporte e o lazer. Belo Horizonte: UFMG, 2008. p. 13-57.

MANHÃES, Eduardo Dias. Políticas de esportes no Brasil. 2.ed. rev. e amp. São Paulo: Paz e Terra, 2002. 286p. 
MEZZADRI, F. M. (Org.). Políticas públicas e esporte. Várzea Paulista: Fontoura, 2014. 288p.

PITTS, B. G. Sport Management at the Millennium: a defining moment. Journal of Sport Management, Champaign, v. 15, p. 1-9, 2001.

SAMPAIO, R. F.; MANCINI, M. C. Estudos de revisão sistemática: um guia para síntese criteriosa da evidência científica. Revista Brasileira de Fisioterapia, São Carlos, v.11, n.1, p. 83-89. Jan./Fev 2007. Disponível em: https://www.scielo.br/pdf/rbfis/v11n1/12 Acesso em: 05 jul. 2016.

SANTOS, A. L. F.; BATISTA, M. C. A.; ARAÚJO, A. V. A produção do conhecimento em política pública para educação física, Esporte e Lazer: o GTT 10 em Pernambuco. In: CONGRESSO BRASILEIRO DE CIÊNCIAS DO ESPORTE [E] II CONGRESSO INTERNACIONAL DE CIÊNCIAS DO ESPORTE / 15, Colégio Brasileiro de Ciências do Esporte. Anais... Recife: CBCE, 2007.

SILVA, A. C. Alguns problemas do nosso ensino superior. Estudos Avançados, v. 15, n. 42, p. 269-93, 2001. Disponível em: https://www.scielo.br/pdf/ea/v15n42/v15n42a14.pdf Acesso em: 05 jul. 2016.

SILVA, E. L. et al. Panorama da Pesquisa em Ciência da Informação. Informação e sociedade: Estudos, João Pessoa, v.16, n.1, p.159-177, Jan/Jun. 2006. Disponível em: https://www.ies.ufpb.br/ojs/index.php/ies/article/viewFile/451/1503 Acesso em: 05 jul. 2016.

SOUZA, C. Políticas Públicas: uma revisão da literatura. Sociologia. v. 8, n. 6, p. 2045, Jul/Dez, 2006. Disponível em: https://www.scielo.br/pdf/soc/n16/a03n16 Acesso em: 05 jul. 2016.

STAREPRAVO, Fernando Augusto. Esporte, política e ciência: a produção científica sobre políticas públicas de esporte e lazer no Brasil. Curitiba: CRV, 2013. 164p.

. Políticas públicas para o Esporte e Lazer: conselhos municipais de Esporte e Lazer e outras formas de participação direta. In: CONGRESSO BRASILEIRO DE CIENCIAS DO ESPORTE [E] II CONGRESSO INTERNACIONAL DE CIÊNCIAS DO ESPORTE. 15, Colégio Brasileiro de Ciências do Esporte. Anais... Recife: CBCE, 2007. p. 1-9.

Políticas públicas de Esporte e Lazer no Brasil: aproximações, intersecções, rupturas e distanciamentos entre os subcampos político/burocrático e científico/acadêmico. Tese (Doutorado em Educação Física). Departamento de Educação Física, Curitiba, UFPR, 2011. 422.f

; MEZZADRI, F. M. Algumas contribuições de Pierre Bourdieu e Norbert Elias à discussão de políticas públicas para o Esporte e Lazer. In: SIMPÓSIO INTERNACIONAL PROCESSO CIVILIZADOR.10, Campinas. Anais... Campinas: 2007. Disponível em $\quad$ https://www.uel.br/grupo- 
estudo/processoscivilizadores/portugues/sitesanais/anais10/Artigos_PDF/Fernando_Au gusto Starepravo>. Acesso em: 05 jul. 2016.

.; SOUZA, J.; MARCHI JÚNIOR, W. Políticas públicas de esporte e lazer no Brasil: uma argumentação inicial sobre a importância da utilização da teoria dos campos de Pierre Bourdieu. Revista Brasileira de Ciências do Esporte, v. 35, n. 3, p. 785-798, 2013. Disponível em: https://revista.cbce.org.br/index.php/RBCE/article/view/743/884 Acesso em: 05 jul. 2016.

STAREPRAVO, Fernando Augusto; SOUZA, J.; MARCHI JÚNIOR, W. A teoria dos jogos competitivos de Norbert Elias como alternativa à leitura das políticas públicas de Esporte e Lazer no Brasil. Revista Brasileira de Educação Física e Esporte (Impresso), v. 26, n. 4, p. 657-665, 2012. Disponível em: https://www.scielo.br/pdf/rbefe/v26n4/v26n4a10.pdf Acesso em: 05 jul. 2016.

- Políticas públicas de Esporte e Lazer no Brasil: uma proposta teóricometodológica de análise. Movimento (UFRGS. Impresso), v. 17, n. 3, p. 233-251, 2011. Disponível em: https://www.seer.ufrgs.br/index.php/Movimento/article/view/18420/14381 Acesso em: 05 jul. 2016.

SCHWARTZ, G. M.; SANTIAGO, D. R. P.; KAWAGUTI, C. N.; TAVARES , G. H.; FIGUEIREDO , J. P.; NAZÁRIO, M. E. S. Gestão da informação sobre esporte recreativo e lazer: balanço da Rede Cedes. Várzea Paulista: Editora, 2010. 192p. Disponível

em: https://www2.esporte.gov.br/arquivos/snelis/esporteLazer/cedes/gestaoInformacao $\% 20$. pdf Acesso em: 07 jul. 2016.

TEIXEIRA, M. G.. IV Plano Diretor para o Desenvolvimento da Epidemiologia no Brasil. Revista Brasileira de Epidemiologia, São Paulo, v. 8, n. 3, p. 231-233, Set, 2005. Disponível em: https://www.scielo.br/pdf/rbepid/v8n3/03.pdf Acesso em: 07 jul. 2016.

WERLE, V. Reflexões sobre a participação nas políticas públicas de Esporte e Lazer. Motriz, Rio Claro, v.16, n.1, p.135-142, Jan/Mar, 2010. Disponível em: $<$ https://www.ufsj.edu.br/portal-repositorio/File/dcefs/Prof._Adalberto_Santos2/7reflexoes_sobre_a participacao_nas politicas_publicas_de esporte e lazer8.pdf $>$ Acesso em: 07 jul. 2016.

ZOTOVICI, S. A.; LOPES, B. R.; RANGEL, R.; STAREPRAVO, F. A.; LARA, L. M. Políticas Públicas de Esporte e Lazer no Brasil e Possibilidades de Intersetorialidade. Licere, v. 16, n. 3, p. 1-31, Set, 2013. Disponível em: $<$ https://www.anima.eefd.ufrj.br/licere/pdf/licereV16N03 re3.pdf $>$ Acesso em: 07 jul. 2016. 
Andrea de O. Barra,

Sheila Aparecida P. Dos S. Silva e

Maria Rachel Vitorino

\section{Endereço das Autoras:}

Andrea de Oliveira Barra

Rua Espírito Santo, 72 - Centro

Varginha - MG - 37.002-240

Endereço Eletrônico: andrea@nepomuceno.cefetmg.br

Sheila Aparecida Pereira dos Santos Silva

Rua Flávio Lazzeti, 53 - Parque dos Príncipes

São Paulo - SP - 05.396-340

Endereço Eletrônico: sheila.silva@uol.com.br

Maria Rachel Vitorino

Rua Lagoa Dourada, 24 - Condomínio Lagoa dos Ipês

Lavras - MG - 37.200-000

Endereço Eletrônico: mrachel1955@gmail.com 